\title{
Modelos de Referencia de Arquitectura Empresarial para la Industria de Educación Superior
}

\section{Enterprise Architecture Frameworks for Higher Education Industry}

\author{
Blanca Lucia Avila Correa ${ }^{1 *}$ \\ Unidad Académica de Tecnologías de Información y Comunicación, Universidad Católica de Cuenca \\ *blavilac@ucacue.edu.ec
}

DOI: https://doi.org/10.26871/killkana_tecnica.v2i1.288

\begin{abstract}
Resumen
La digitalización de las universidades es uno de los grandes desafíos de la universidad ecuatoriana en la actualidad. La búsqueda de la calidad en la educación superior implica el logro de transformaciones desde la perspectiva estratégica, por lo que los líderes de TI deben estar preparados para formar parte del equipo de toma de decisiones que entienda las necesidades empresariales, proponga soluciones tecnológicas que garanticen la generación de valor. A lo largo del tiempo, la falta de entendimiento de las necesidades de los grupos de interés, ha llevado a proponer soluciones aisladas y desconectadas, que han abordado los problemas sin hacer un análisis causal. El presente trabajo, busca motivar a los líderes de TI de las instituciones de educación superior del Ecuador la implementación de procesos de arquitectura empresarial a plantear soluciones estratégicas y aborden la problemática de las universidades de forma integral, articulando los componentes estratégicos, del negocio, de las aplicaciones, datos y la tecnología a través de arquitectura empresarial. La propuesta se basa en el marco de referencia TOGAF y su metodología ADM, a la que se suman una lista de marcos de referencia, modelos, estándares y buenas prácticas alineadas a la educación superior. Estos referentes deberán ser adoptados en función del contexto la necesidad de cada institución. en el entorno en el que se aplican. Cabe mencionar que en este trabajo se han omitido otras herramientas y métodos propios del marco de referencia, los cuales deberán ser adoptados en el ejercicio de la arquitectura empresarial.
\end{abstract}

Palabras clave: Área de la Organización, Dominio, Iniciativa, Problema, Marco de referencia.

\begin{abstract}
The digitalization of the universities is one of the great challenges of the Ecuadorian university at present. The search of the quality in the higher education implies the achievement of transformations from the strategic perspective. For that reason, the CIOs must be prepared to take part to the decision-making team, which understands the business needs, proposes technological solutions that guarantee the generation of value.Over time, the lack of understanding of the needs of stakeholders, has led them to propose isolated and disconnected solutions, which have addressed the problems without doing a causal analysis. The present work seeks to motivate to CIOs of the higher education institutions of Ecuador to implement business architecture to propose strategic solutions and address the problems of the universities in an integral manner, articulating the strategic components of the business, Applications, data and technology through business architecture. The proposal from the TOGAF reference framework and its ADM methodology is add a list of reference frameworks, models, standards and good practices aligned to higher education. These referents should be adopt depending on the context the need of each institution in the environment in which they are applied. It is worth mentioning that in this work other tools and methods of the frame of reference have been omit, which should be adopt in the exercise of the business architecture.
\end{abstract}

Key words: Enterprise, Domain, Initiative, Concern, Framework.

\section{INTRODUCCIÓN}

El relevante papel de las TICs en las organizaciones, exige que los departamentos de TI propongan soluciones tecnológicas que logren una verdadera transformación estratégica.

La consolidación de las organizaciones, requiere solu- ciones estratégicas de tecnología, con equipos gerenciales que tengan un alto nivel de liderazgo; entonces, el líder de TI, debe formar parte del equipo de toma de decisiones y lograr resultados que generen valor al negocio y resuelvan problemas empresariales. Desde esta perspectiva, el líder de TI debe ser un estratega; que entienda las necesidades 
empresariales, proponga soluciones y plantee alternativas de logro.[1]

Puesto que la Arquitectura Empresarial es la disciplina encargada de entregar soluciones a los problemas empresariales; debe satisfacer las necesidades empresariales de los grupos de interés (accionistas, empleados, clientes, proveedores, entidades regulatorias, etc.). Entendiéndose por necesidades empresariales al incremento de la rentabilidad, de la satisfacción de los clientes, la reducción de costos o la optimización de las inversiones.

Entonces, ¿Cómo puede TI entender todas estas necesidades, traducirlas en procesos de negocio, mantener el modelo jerárquico dentro de la empresa y buscar que estos procesos tengan soluciones tecnológicas, innovadoras, sostenibles, estructuradas e integrales?; la respuesta está en la implementación de Arquitectura Empresarial.

Ahora, ¿Cómo reconocemos que una organización requiere implementar trabajos de arquitectura empresarial? Debido al incesante cambio que sufren muchas empresas, su adaptación al entorno dinámico requiere desarrollar un sentido de urgencia, que contrarreste con esta realidad. Esto frecuentemente termina haciendo que TI proponga soluciones tecnológicas aisladas y temporales. Por otro lado, la complejidad y la premura convergen en una necesidad de priorización y jerarquización de los problemas estratégicos, tácticos y operativos. Su desarticulación, produce soluciones aisladas y el desconocimiento de la problemática por parte de los niveles operativos, haciendo que la organización se desgaste en soluciones que no agregan valor.[1]

Para ilustrar cómo las organizaciones pueden consolidarse a través de los procesos de Arquitectura Empresarial, el presente trabajo pretende orientar al lector sobre el proceso de arquitectura empresarial con enfoque en la educación superior. La elección de este sector se hace en función de los innumerables cambios a los que se han sometido las instituciones de educación superior en el Ecuador desde el año 2010, luego de que entrara en vigencia la Ley de Educación Superior (LOES), además de la dinámica de las tendencias tecnológicas cuya adopción las vuelve altamente competitivas y sostenibles. En este contexto, el trabajo se enfocará en puntualizar el proceso y los referentes más relevantes para el desarrollo de una arquitectura empresarial en las universidades.

\section{FUNDAMENTOS DE LA ARQUITECTURA EMPRESARIAL}

La arquitectura empresarial, se ha consolidado como una propuesta de solución de problemas empresariales que abordan la raíz de los problemas de manera estructurada para encontrar soluciones definitivas apoyadas en tecnología.

En muchas organizaciones, el entendimiento a la problemática empresarial es sintomático; es decir, no se hace un análisis causal antes de definir un esquema de solución. La arquitectura empresarial fue creada para que los responsables de TI puedan erradicar los problemas haciendo una interpretación de la realidad a través del análisis causal y proponiendo soluciones preventivas, proactivas y predictivas. [1]

En el caso de las universidades del Ecuador, los factores de sostenibilidad y competitividad, crean la necesidad urgente de apalancamiento de los procesos académicos, de investigación y de vinculación con la sociedad; con soluciones tecnológicas estratégicas que reduzcan la abrumadora brecha de transformación digital que tienen respecto las exigencias del mercado internacional. Las tendencias tecnológicas están propuestas en los informes del Espacio Europeo de Educación Superior (EEES), el informe Horizon del New Media Consotium, los informes anuales de EDUCAUSE y Gartner entre otros.[2]

\section{A. Dominios de la Arquitectura Empresarial}

Cuando hacemos un visionamiento del ecosistema institucional de las universidades, al igual que en otras organizaciones se evidencian componentes estratégicos, de negocio y tecnológicos. Precisamente, desde esta perspectiva, la arquitectura empresarial enfoca el tratamiento de las soluciones empresariales mediante la articulación de las estrategias, los componentes de negocio (procesos y organización), la información, las aplicaciones, la infraestructura tecnológica y los riesgos y seguridades.[3]

Todos los marcos de trabajo de la arquitectura empresarial, abordan de una u otra forma estos componentes a los que se denominan Dominios de la Arquitectura Empresarial y su clasificación puede variar. Por ejemplo; para el padre de la arquitectura empresarial, John Zachman; esta disciplina se consolida en cinco dominios: Estrategias y Normatividad, Estructura, Procesos de Negocio, Información y Servicios de TICs; mientras que en el marco de referencia TOGAF (The Open Group Architecture Framework), éstos se agrupan en cuatro dominios: Negocio, Aplicaciones, Información y Tecnología. Este último enfoque, será tratado en el presente trabajo.[2]

De cualquier modo, la articulación de estos dominios, establece un lenguaje común de entendimiento, para que cada uno genere valor a la organización.

\section{Dominio del Negocio}

En este dominio se definen los componentes estratégicos y del negocio. El análisis de los componentes estratégicos comprende: los grupos de interés, los elementos de direccionamiento estratégico (misión, visión, políticas, diagnóstico situacional, mapa estratégico y entorno organizacional) el análisis competitivo, los servicios del negocio y el modelo de negocio.[4]

En el análisis del negocio se deben articular; la cadena de valor, los procesos estratégicos de apoyo; así como la organización, sus roles y competencias.[5]

\section{Dominio de Aplicaciones}

Consiste en el análisis de las plataformas de Core, las aplicaciones existentes y requeridas por la organización. De cada aplicación debe especificarse un plano de los sistemas 
y sus interrelaciones con los procesos más importantes y la información que gestionan.[5]

Es importante que este dominio logre que la organización alinee sus aplicaciones con las necesidades del negocio. Para ello se deberá identificar cuáles son las brechas entre la arquitectura de aplicaciones actual y la propuesta, a través de una evaluación de las capacidades, un plan de comunicación y el modelo organizacional.

\section{Dominio de Información}

Consiste en la descripción de los datos asociados a con las aplicaciones. Es decir, los datos de los macro-procesos que son parte de los datos corporativos y que apoyan la gestión a través de bases de datos, sistemas de gestión de bases de datos, repositorios de información, etc. Adicionalmente se analizarán los métodos de administración, los flujos de datos, los modelos de análisis de desempeño y toma de decisiones, los maestros de datos y las políticas de seguridad de la información.[6]

Es necesario que, en este dominio se consideren aspectos anclados a los sistemas de aplicación que los procesan, cómo se relacionan entre sí y la relación de los sistemas con los procesos que los gobiernan.

\section{Dominio de Tecnología}

En este dominio se debe describir la situación tecnológica actual respecto al software de base, la infraestructura física, las redes y telecomunicaciones; así como los componentes de seguridad requeridos.[6]

El análisis debe considerar cuáles son las necesidades de nuevas soluciones tecnológicas. Cada uno de los componentes debe contener una arquitectura actual y propuesta que se derive de las brechas encontradas para ser analizadas.[2]

\section{B. Proceso de Arquitectura Empresarial para las Institu- ciones de Educación Superior}

El proceso de arquitectura empresarial se basa en la taxonomía de los componentes de los dominios y la ontología entre los objetivos estratégicos de negocio con los componentes tecnológicos de cada dominio.[7]

Para abordar la complejidad de las organizaciones mediante la articulación de los dominios; es necesario guiar el ejercicio de arquitectura empresarial con un marco de trabajo que establezca los lineamientos, metodología y técnicas.

Los marcos de referencia para el desarrollo de la arquitectura empresarial más comunes son: Zachman, FEA (Federal Enterprise Architecture), Gartner y TOGAF.[8]

El marco de referencia de Zachman concibe el desarrollo de la arquitectura empresarial como una desagregación de los componentes empresariales tales como: estrategias, procesos, organización, información, aplicaciones e infraestructura. Esta organización permite responder al qué, cómo, dónde, quién, cuándo y el porqué de un proyecto en cada dominio. El proceso taxonómico define hasta 36 subniveles de estratificación de los dominios. [8]

El marco de referencia FEA (Federal Enterprise Architecture), propuesto por el gobierno de los Estados Unidos, está centrado en describir y analizar las inversiones de TI, mejorar la colaboración y transformar el gobierno para el desarrollo de la arquitectura empresarial. Éste marco de referencia relaciona el rendimiento, negocio, servicios, datos para establecer la alineación estratégica entre el negocio y la tecnología.[9]

El marco de referencia de Gartner se enfoca en el incremento de la rentabilidad a través de la agrupación de los propietarios del negocio, los especialistas de la información y los implementadores de tecnología. Este modelo utiliza la metodología GEAM (Gartner Enterprise Architecture Method) para fusionar la arquitectura del negocio, la arquitectura de las aplicaciones y datos y la arquitectura de tecnologías en el contexto organizacional.[10]

La propuesta del Open Group, para el desarrollo de la Arquitectura Empresarial es TOGAF. Es considerado por los especialistas como un referente agnóstico y está disponible de forma libre en la web. [2] Estas características favorecen la implementación de la arquitectura empresarial en las instituciones de educación superior del Ecuador, por lo que será explicado con mayor detalle.

TOGAF fundamenta el trabajo de arquitectura empresarial en los dominios del negocio, información, aplicaciones y tecnología. Se basa en el ADM (Architecture Development Method), el cual describe el procedimiento que va desde el entendimiento de una necesidad de Arquitectura Empresarial hasta la solución del problema empresarial (concern) [11]. El ADM de TOGAF se define en diez fases (figura 1.), cada una de las cuales provee al arquitecto empresarial; la guía de desarrollo de la arquitectura empresarial, los entregables, artefactos y la validación de los resultados. Este método es iterativo en cada proceso de arquitectura empresarial y entre sus fases lo que permite avanzar de forma sistemática e incremental o incluso volver al punto de partida.

\section{Fase Preliminar}

La fase preliminar es la etapa de alistamiento de la arquitectura empresarial, en ésta se resuelve qué tan preparada está la organización para acoger un ejercicio de arquitectura empresarial. Es decir, cuál es la capacidad de la empresa para realizar la arquitectura empresarial. Para ello se debe determinar; dónde, qué, porqué, quién y cómo se va a desarrollar la arquitectura empresarial[11]. Esto significa definir el contexto o área de la organización a considerar (enterprise), cuál es el problema (concern), los principios o reglas a corto plazo que regirán el proceso, el gobierno y el equipo de trabajo que formulará la arquitectura; además de las técnicas y guías que apoyarán el proceso.

Quienes lideran el proyecto, deberán estructurar y desarrollar todos los elementos que se relacionen con los antecedentes y el contexto de la empresa, la metodología y la toma 
de decisiones informadas sobre la aplicación del marco de trabajo.

La identificación de los grupos de interés y sus necesidades, un plan de riesgos, el modelo de negocio que identifique; los objetivos estratégicos, la cultura organizacional y jerarquía en la toma de decisiones; los procesos actuales documentados, las metodologías de gestión, el portafolio de proyectos, el mapa de ruta con el presupuesto alineado entre otros; deben estar disponibles antes de arrancar el proceso de arquitectura empresarial.

Fig. 1. Esquema de la Metodología ADM de TOGAF

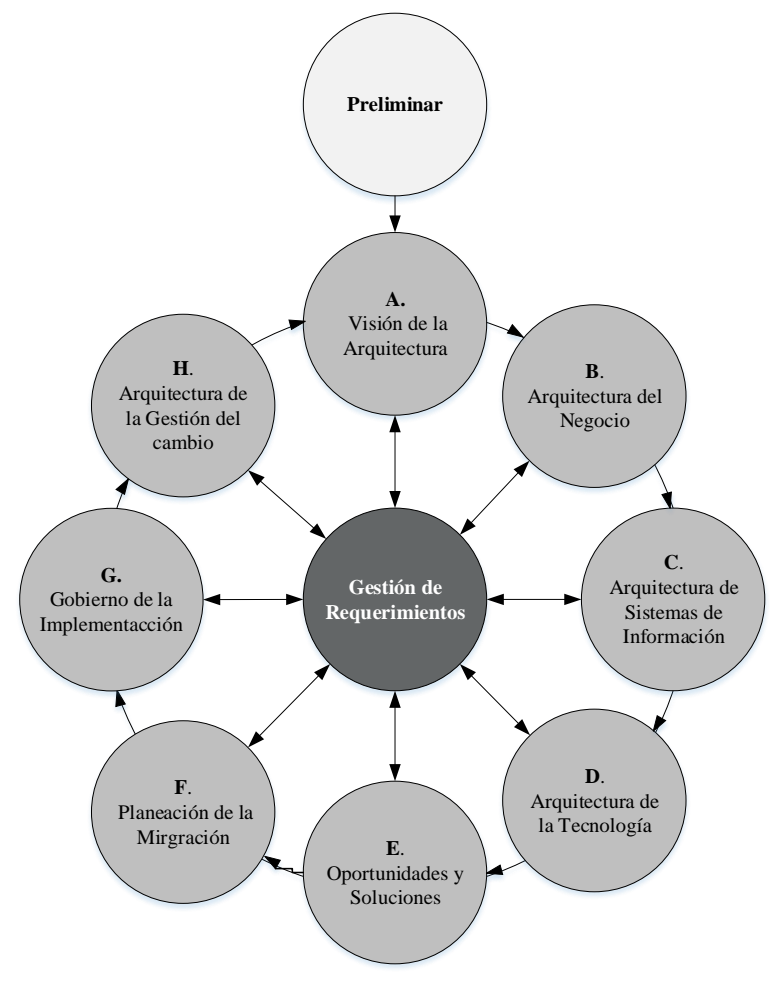

Fuente: The Open Group

\section{Fase A: Visión de la Arquitectura}

La fase visionamiento de la arquitectura, consiste en definir un escenario de llegada de la arquitectura; es decir, cuáles son las capacidades que se quieren desarrollar. Se trata de obtener una vista panorámica a través de un reconocimiento de alto nivel que incluya todos los componentes estratégicos y de dirección de la institución que respondan a los motivadores de negocio, nuevos escenarios y cambios de normativas.

En esta fase el equipo de arquitectura debe considerar evaluar la misión, visión, valores, gobierno, estrategias tanto del negocio como de TI; analizar la situación actual mediante FODA (Fortalezas, Oportunidades, Debilidades y Amenazas) o ERIC (Eliminar, Reducir, Incrementar o Crear); analizar la competencia, la competitividad, los grupos de interés, el portafolio de productos y servicios; definir el modelo de negocio y los requerimientos funcio- nales y no funcionales que apoyan la formulación de la arquitectura[11].

\section{Fase B: Arquitectura del Negocio}

En esta fase corresponde definir una línea de partida, línea base o Base Line de los componentes del negocio. Ello implica definir los procesos, servicios y funciones.

En este punto, el equipo de arquitectura empresarial debe determinar los motivadores o razones que apalancan el desarrollo de la arquitectura, analizar la organización (organigrama institucional), funciones, personas y roles institucionales, así como sus inter-relaciones. Analizar el mapa de procesos institucional con el detalle de los procesos estratégicos y misionales (académicos, de investigación y de vinculación con la sociedad) y los de soporte.[2]

Asimismo, será necesario determinar los productos o servicios, como la oferta académica de pregrado, posgrado, las diferentes modalidades de estudio entre otros.[2]

\section{Fase C: Arquitectura de los Sistemas de Información}

En este punto del proceso, el equipo deberá usar esquemas de arquitectura que definan la línea base de las aplicaciones clave que gestionan los procesos institucionales, tales como plataformas de Core, de servicios, interfaces y canales, aplicaciones móviles, aplicaciones para la toma de decisiones, plataformas de integración y bus de servicios, etc.[12] Además se debe establecer su relación con los procesos para visualizar necesidades de implementación.

De igual manera, el equipo de arquitectura empresarial, deberá definir esquemas de arquitectura que identifiquen los activos de información institucionales como; bases de datos relacionales, no relacionales, su administración y consistencia, los repositorios de contenidos digitales, modelos de análisis de desempeño de inteligencia de negocios y toma de decisiones, datos maestros institucionales, sistemas de gestión de seguridad informática. La profundidad del análisis deberá contemplar las relaciones de éstos con las aplicaciones que los gestionan.[2]

\section{Fase D: Arquitectura de la Tecnología}

La definición de la línea base de la infraestructura tecnológica deberá hacerse especificando los componentes tecnológicos y los equipos de seguridad, la infraestructura de redes y comunicaciones de amplia cobertura, las redes locales e inalámbricas, la infraestructura de almacenamiento y los centros de datos, los clústeres de virtualización, y el software de bases de datos. En este último se deberán incluir el licenciamiento, niveles de estandarización y principalmente las políticas de seguridad.[2]

En cada uno de los modelos arquitectónicos de línea base definidos en las fases, B, C, y D; deberá existir una arquitectura propuesta de solución que determina hacia dónde se quiere llegar; es decir, la arquitectura objetivo o target. Esta propuesta debe estar basada en las tendencias, estándares, modelos, marcos de referencia y mejores prácticas propias de la industria de la educación superior. Este escenario, 
debe asegurar el cumplimiento de los requerimientos de los grupos de interés y los problemas identificados.[12]

Sobre esta base, lo siguiente es establecer las diferencias entre el escenario actual y el futuro (baseline y target) para definir y analizar las brechas de cada uno de los elementos de los dominios de la arquitectura empresarial. Una de las herramientas más usadas en este punto es el Modelo de Madurez de las Capacidades que permite evaluar cada componente en una escala que va de 0 (incompleto) a 5 (optimizado).[2] En función del resultado de la evaluación se define el escenario target, el cual representa el referente más próximo para logar un cambio significativo.

Del GAP Analysis o análisis de brechas, se desprenden las iniciativas de solución que pasarán a formar parte de una hoja de ruta o Roadmap.[11]

\section{Fase E: Oportunidades y Soluciones}

Las brechas detectadas representan las necesidades, insuficiencias o carencias que tienen la institución; es decir las iniciativas. La agrupación lógica de iniciativas conformará los paquetes de trabajo de arquitectura empresarial. Aquí, se afinan detalles del Mapa de Ruta de manera que se convierta en una herramienta estratégica de conducción de los paquetes de arquitectura empresarial hacia la consecución de las necesidades de los grupos de interés. Las soluciones y oportunidades definidas, deberán también contemplar las limitaciones de ejecución. [11]

Las iniciativas empaquetadas, deberán seleccionarse en base a los motivadores del negocio, los cuales generalmente buscan incrementar la rentabilidad, la satisfacción de los clientes, reducir los costos u optimizar las inversiones.

La versión completa del mapa de ruta, deberá incorporar los costos, el tiempo, los recursos y un análisis de las implicaciones que tendrá la implementación de las arquitecturas. Este mapa de ruta representa el plano de la capacidad de transición de la arquitectura y deberá estar acompañado de un plan de implementación y migración.

\section{Fase F: Planeación de la Migración.}

La fase anterior, define la capacidad de la institución para la transición de la línea base a la arquitectura objeto, considerando la relación costo-riesgo-beneficio. En este punto, el equipo de arquitectura empresarial, debe detallar el plan de implementación y migración para hacer realidad la arquitectura target definiendo entregables, hitos y listas de verificación que permitan hacer un seguimiento y monitoreo del proceso. Este plan debe estar coordinado con los procesos de gestión del portafolio y de proyectos de la institución. Asimismo, deberá considerar aspectos de gestión del cambio que incluyan actividades de evaluación de dependencias, costos y beneficios de cada proyecto en el contexto institucional. [11]

\section{Fase G: Gobierno de la Implementación}

El Plan de Migración e Implementación, se elabora por la necesidad de supervisar la implementación de acuerdo a lo establecido en él. El cumplimiento de este plan requiere de la definición de Acuerdos de Niveles de Servicio (SLA) y una adecuada Gestión de Proveedores. [2]

Para reducir los riesgos del fracaso en la implementación de los paquetes de arquitectura, deberá supervisarse exhaustivamente. De esto se encarga la función de Gobierno de la Arquitectura. El aseguramiento de la implementación, implica que ésta se realice con una serie de transiciones incrementales hacia la arquitectura deseada. [11]

\section{Fase H: Gestión de Cambio de la Arquitectura}

El objetivo de esta fase es cumplir con el proceso de mejora continua a través de la evaluación constante del trabajo de arquitectura, que detecte resultados desfavorables en los ciclos de implementación de la arquitectura.[1]

Cada ejercicio de arquitectura, deberá estar acompañado de una adecuada gestión del cambio que garantice el cumplimiento de la arquitectura deseada que apunta a agregar valor al negocio. En este punto es necesario que el equipo de arquitectura considere alternativas de roll back que permitan regresar a un estado anterior de ser necesario. [11]

La gestión del cambio, se encargará de supervisar continuamente nuevas solicitudes, desarrollos tecnológicos o cambios del entorno empresarial. Una vez identificado el cambio, el proceso de gestión de cambio determinará si es necesario iniciar formalmente un nuevo ciclo evolutivo de la arquitectura y permitirá establecer criterios para juzgar si una solicitud de cambio requiere actualización o necesita un nuevo ciclo.

\section{MARCOS DE REFERENCIA COMPLEMENTARIOS PARA EL TRABAJO DE LA ARQUITECTURA EMPRESARIAL EN LA EDUCACIÓN SUPERIOR}

Los marcos de referencia de la arquitectura empresarial, guían el trabajo para llevar a las organizaciones a un escenario deseado. Este trabajo se ejecuta con el apoyo de estándares, modelos, marcos de referencia y buenas prácticas propios de cada vertical de negocio.

Las instituciones de educación superior, por naturaleza, tienen componentes, organizaciones y procesos complejos que han llevado a varios organismos y firmas internacionales a proponer metodologías, técnicas y herramientas que pueden ser aplicados durante el proceso de arquitectura empresarial.

BMM (Business Motivation Model), usado con frecuencia en los ejercicios de arquitectura empresarial para justificar las razones por las que se debe realizar el trabajo. Permite la planeación de las iniciativas y la evaluación rigurosa de los resultados. [2]

El Análisis de las Cinco fuerzas Competitivas de Porter, es un referente que apoya el análisis de la competitividad. Las universidades pueden hacer uso de esta herramienta para definir su posición frente a la competencia y hacer frente a ella asegurando su sostenibilidad.[13] 
La Matriz BCG (Boston Consulting Group), permite a las universidades autofinanciadas definir un portafolio de productos o servicios que generen mayor valor. BCG optimiza la oferta académica, a través de un reconocimiento de los niveles de crecimiento y participación del mercado. Aquellas que tienen bajos niveles de participación o crecimiento, representan un problema para la universidad.[14]

APQC es un referente que proporciona la taxonomía para la estandarización de los procesos de diversas industrias, entre las que figura la educación superior. La estandarización de los procesos facilita la evaluación comparativa (benchmarking) para un análisis y debate objetivo de las actividades.[15]

IWA2:2007 (International Workshop Agreement), es un marco de referencia orientado a la gestión de la calidad de la educación superior. Basada en ISO 9001:2000.[16]

PMBOK (Project Management Body of Knowledge), orientado a la gestión de proyectos, debe ser usado en los ejercicios de arquitectura empresarial de las universidades para gestionar las iniciativas seleccionadas.[17]

PRINCE 2 (Projects in Controlled Environments), apto para la gestión de iniciativas de arquitectura empresarial, provee un conjunto de buenas prácticas para la gestión de proyectos con un manejo de altos niveles de variabilidad e incertidumbre en entornos controlados.[18]

CMM (Capability Maturity Model), es un marco de referencia encargado de la evaluación de los procesos. Es usado para definir el grado de madurez de los componentes de los dominios de arquitectura empresarial en una escala de 0 (inexistente) a 5 (implementado y maduro) y la consecuente identificación de brechas. [2]

La Gestión Estratégica de Proveedores, permite la administración y evaluación del rendimiento de los proveedores, a través de la aplicación de mejores prácticas, control de gasto, reducción de riesgos y aprovecha de la generación de valor para el negocio.

El informe anual de Tendencias Mundiales de Capital Humano de Deloitte, es una guía que las universidades pueden adoptar para la definición de una estructura organizacional de vanguardia, cuyas estrategias de gestión de capital humano se alineen con la disrupción digital.[19]

A estos referentes se suman otros propios de la gestión de TI tales como:

PETI para la Planeación estratégica de TI que cubra las necesidades de los niveles; estratégicos, directivos y operativos de la institución y garantice el adecuado uso de los recursos, las inversiones tecnológicas y el proceso de implementación de las soluciones tecnológicas. El PETI permite que los responsables de TI cuenten con estrategias de TI embebidas en las estrategias de negocio y realizar la toma de decisiones respecto a las inversiones tecnológicas, la capitalización las innovaciones, la implementación de soluciones de economía de escala, etc. [2]

El marco de referencia COBIT (Control Objetives for Information and related Technology) apoya a los líderes de TI de las instituciones educativas con las mejores prácticas de control y supervisión apuntando a satisfacer las necesidades de los grupos de interés y la generación de valor.[20]

La Biblioteca de Infraestructura de Tecnologías de Información ITIL (Information Technology Infrastructure Library), proporciona las mejores prácticas en la gestión de servicios de TI y define acuerdos de niveles de servicio SLA con proveedores de servicios tecnológicos. Los responsables de TI de las instituciones de educación superior pueden adoptar esta guía para: Gestionar Incidencias, Problemas, Cambios, Configuraciones y Versiones de las soluciones tecnológicas y los servicios que nacen de las iniciativas de arquitectura empresarial. [2]

El uso de la arquitectura SOA (Service Oriented Architecture) en las instituciones de educación superior, apoyan la implementación de un esquema de interacción e integración con los sistemas de información de externas[21] como CEAACES, SENESCYT, etc. Este modelo propicia la accesibilidad de las aplicaciones web y contenido públicos propio de estas instituciones.

Las universidades al igual que otras organizaciones deben tener una gestión eficiente de sus activos de información. DAMA-DMBOOK es un marco de referencia que proporciona las directrices para la gestión eficiente de la información.[22]

Finalmente, para la protección de datos y seguridades de la institución, debe aplicarse el estándar ISO 27002-2013 para implementar el Sistema de Gestión de las Seguridades de Información. Este estándar proporciona las normas para la selección, implementación y administración de controles de la seguridad de la información y las mejores prácticas de la gestión de la seguridad de la información.[23]

\section{CONCLUSIONES}

La solución de problemas empresariales a través de los procesos de Arquitectura Empresarial en el Ecuador es relativamente nueva si lo comparamos con países de la región como Colombia y Costa Rica; por lo tanto, el aporte de este trabajo radica en promover en las universidades del Ecuador implementaciones de arquitectura empresarial.

La adopción de soluciones pertinentes a través de arquitectura empresarial en las universidades ecuatorianas, radica fundamentalmente en el exigente y complejo entorno actual debido a los altos niveles de competitividad, la necesidad de sostenibilidad, las exigencias de los organismos rectores y las tendencias mundiales de la digitalización.

La descripción del proceso de arquitectura empresarial está fundamentada en el marco de referencia TOGAF propuesto por el Open Group. Su elección se basa en el estudio comparativo realizado por Robert Sessions en el que TOGAF obtiene una importante puntuación en la Integridad del proceso, neutralidad del proveedor y la disponibilidad de información. Su ventaja frente a FEA, Zachman y Gartner rase debe a los costos de implementación. [2]

Los marcos de referencia complementarios de este trabajo son propuestos a modo de sugerencia. El amplio abanico de posibilidades de buenas prácticas, normativas y modelos 
del mercado dejan en libertad de elección a los responsables de la arquitectura empresarial. Sin embargo, en este trabajo se ha procurado seleccionar los referentes que mejor se orientan a la vertical de la educación superior.

Los marcos de referencia complementarios propuestos en el presente trabajo, se correlacionan con las actividades de arquitectura empresarial en los dominios; del negocio, aplicaciones, datos y tecnología y se deben proponer en el mapa de ruta detallado de la fase $E$ de TOGAF

\section{REFERENCIAS}

[1] J. Monsalve, "Referencia de Arquitectura Empresarial," 2015.

[2] B. Avila, Formulación de Visionamiento Estratégico de Arquitectura Empresarial aplicado a Entidades de Educación Superior: Un Caso de Aplicación. PhD thesis, 2016.

[3] M. Darío Arango Serna, J. Enrique Londoño Salazar, and J. Andrés Zapata Cortés, "ARQUITECTURA EMPRESARIAL - UNA VISIÓN GENERAL," $R e$ vista Ingenierías Universidad de Medellín, vol. 9174, no. 16, 2010.

[4] "Business Strategy - Enterprise Architecture."

[5] Wikidot, "Application Architecture - Enterprise Architecture."

[6] Wikidot, "Technology Architecture - Enterprise Architecture."

[7] C. O'Rourke, N. Fishman, and W. Selkow, Enterprise architecture using the Zachman Framework. Course Technology, a division of Thomson Learning, 2003.

[8] J. Schekkerman, How to survive in the jungle of enterprise architecture frameworks : creating or choosing an enterprise architecture framework. Trafford, 2004.

[9] "Federal Enterprise Architecture Framework," 2013.

[10] R. S. Bittler and G. Kreizman, "Gartner Enterprise Architecture Process: Evolution 2005," Gartner Enterprise Architecture Process, no. October, p. 12, 2005.

[11] The Open Group, TOGAF Versión 9.1. 2011.

[12] J. Laviña Orueta dir., L. Mengual Pavón dir., and F. Telefónica, Libro blanco de la universidad digital 2010. 2008.

[13] M. E. Porter and M. E. Rosas-Sánchez, Estrategia competitiva : técnicas para el análisis de los sectores industriales y de la competencia. Continetal, 2000.

[14] T. d. Marmol, BCG growth-share matrix.

[15] APQC, "Education Process Classification Framework 7.0.5," 2016.

[16] IWA, "Guía de aplicación -IWA 2 - Sistemas de getión de la calidad para organizaciones educativas," pp. 1100, 2002.

[17] Project Management Institute, PMBOK: Guía de Fundamentos de Dirección de Proyectos. 2013.

[18] O. o. G. Great Britain. Office of Government Commerce. and O. o. G. Commerce, Managing successful projects with PRINCE2. TSO, 5th ed., 2009.
[19] Deloitte, "Tendencias Mundiales de Capital Humano 2017."

[20] ISACA, COBIT 5 - Un Marco de Negocio para el Gobierno y la Gestión de TI de la Empresa. 2012.

[21] I2B, “QQué se entiende por SOA, y cuáles son sus beneficios? I I2B Intelligence to Business."

[22] M. Mosley, "DAMA DMBOK Functional Framework," Dama-Dmbok, vol. 3.02, pp. 1-19, 2008.

[23] ISO, "ISO 27002 en español," Normativa ISO, vol. 2013, 2013.
Recibido: 3 de mayo de 2018

Aceptado: 15 de junio de 2018 
